\title{
Effects of stubborn decision-makers on vaccination and disease propagation in social networks
}

\author{
Eriko Fukuda* and Jun Tanimoto \\ Interdisciplinary Graduate School of Engineering Sciences, \\ Kyushu University, \\ Kasuga-koen, Kasuga-shi, \\ Fukuoka 816-8580, Japan \\ Email: eriko.fukuda@kyudai.jp \\ Email: tanimoto@cm.kyushu-u.ac.jp \\ *Corresponding author
}

\begin{abstract}
In this study, we investigate how the presence of 'stubborn' individuals affects other individuals' vaccination behaviour with regard to the spread of infectious disease in a lattice populations and a Barabási-Albert scale-free network. (In this context, 'stubborn' individuals are those who are intransigent about their own vaccination strategy). To meet this research objective, we develop a combined model that is constructed from epidemiological and vaccination dynamics and is based on adaptive imitation behaviour. With regard to preventing disease propagation, the stubborn vaccinated individuals act as 'good role models', as they always take vaccines, whereas the stubborn unvaccinated individuals, in contrast, act as 'bad role models', as they always refuse vaccines. As a result, which stubborn individuals are more influential among the full population in terms of voluntary vaccination behaviour depends not only on their proportion within the population, but also the network structure and the cost of vaccination.
\end{abstract}

Keywords: vaccination decision; mathematical epidemiology; evolutionary game theory; vaccine-preventable diseases.

Reference to this paper should be made as follows: Fukuda, E. and Tanimoto, J. (2016) 'Effects of stubborn decision-makers on vaccination and disease propagation in social networks', Int. J. Automation and Logistics, Vol. 2, Nos. 1/2, pp.78-92.

Biographical notes: Eriko Fukuda is a PhD student at the Interdisciplinary Graduate School of Engineering Sciences, Kyushu University. Her current research interests include complex systems, epidemiology, and evolutionary games.

Jun Tanimoto is a Professor at Kyushu University. He obtained his $\mathrm{PhD}$ in engineering from Waseda University, and his current research interests include complex science, evolutionary games, traffic flows, urban climatology, and building physics.

This paper is a revised and expanded version of a paper entitled 'Impact of stubborn individuals on a spread of infectious disease under voluntary vaccination policy' presented at The 18th Asia Pacific Symposium on Intelligent and Evolutionary Systems (IES 2014), Singapore, 10-12 November 2014. 


\section{Introduction}

In 1918, the Spanish flu produced the most serious pandemic in recorded history; the Asian flu (1957), the Hong Kong flu (1968), and the swine flu (2009) have been the most recent pandemics. In humankind's long history, the spread of global infectious diseases such as the flu has remained a serious threat. Much effort has been devoted, over an extended period, to modelling and analysing the spread of global infectious diseases, with an eye to controlling and preventing it (Anderson and May, 1991; Bailey, 1975).

Pre-emptive vaccination is one of the most powerful public health measures by which to immunise people against vaccine-preventable diseases like influenza, measles, and chickenpox (Anderson and May, 1991), and people are basically expected to get themselves voluntarily vaccinated for such infectious diseases. Under a voluntary vaccination policy, an individual's decision-making attitude vis-à-vis vaccination is determined by several factors, such as perceived risks of infection and vaccination, self-interest, religious reasons, and the vaccination response behaviour of others (neighbours), inter alia (Basu et al., 2008; Bauch et al., 2003; Bauch and Earn, 2004; Bauch, 2005; Bauch et al., 2007; Chapman and Coups, 1999; Chapman and Coups, 2006; Funk et al., 2010; Schimit and Monteiro, 2011). When the vaccination level within a population is increased and it exceeds a critical level, herd (community) immunity which prevents an epidemic from spreading further in the population - is attained (Anderson and May, 1991). Consequently, the herd immunity as public goods protects unvaccinated individuals indirectly. As a result, unvaccinated individuals escape infection without incurring any of the possible risks imposed by vaccination (e.g., complications, side effects, and economic cost) (Anderson and May, 1991; Brisson and Edmunds, 2003). As a result, some individuals may be tempted to take a 'free ride' on this benefit - in other words, it decreases their incentive to get vaccinated. This is the well-known dilemma with respect to vaccination that leads to conflict between the rational decision-making of each individual and the optimal vaccination level for the whole of society (i.e., the aforementioned critical level for herd immunity). Some reports indicate that this policy makes it very difficult or even impossible, in the absence of other incentives, to completely eradicate a vaccine-preventable disease (Bauch et al., 2003; Bauch and Earn, 2004; Bauch et al., 2007; Vardavas et al., 2007).

To characterise the decision-making process of individuals and the vaccination dilemma itself, in recent years, some researchers have studied the voluntary vaccination behaviour of individuals who face epidemics. Many of these studies use an evolutionary game theoretic framework in epidemiologic dynamics, or the so-called vaccination game (Bauch, 2005; Bauch and Bhattacharyya, 2012; Fu et al., 2011; Fukuda et al., 2014; Liu et al., 2012; Ndeffo Mbah et al., 2012; Perisic and Bauch, 2009; Vardavas et al., 2007). For example, Bauch (2005) constructed a combined framework where epidemiological dynamics is inserted into the imitation (learning) dynamics of vaccination behaviour, based on evolutionary game theory; he found that an individual's imitation behaviour with respect to vaccination can lead to oscillations in disease outbreaks. Early studies with regard to the vaccination game have not taken into account the network structure where diseases propagate in the population - that is, the population has been assumed to be well-mixed. However, in reality, any network has a certain structure. Therefore, much researchers have focused on the disease propagation in social (contact) networks, inter alia (Keeling and Eames, 2005; Meyers et al., 2005; Pastor-Satorras and Vespignani, 
2001). Accordingly, Fu et al. (2011) studied the impact of population structures on voluntary vaccination behaviour, based on imitation dynamics; they found that the population structure acts as a 'double-edged sword' for public health - namely, it depends largely on the cost of vaccination in promoting individual vaccination behaviour. Ndeffo Mbah et al. (2012) examined the impact of imitation behaviour with vaccinations in social contact networks. They asserted that imitation behaviour may help impede the eradication of infectious diseases, because such behaviour leads to clusters of susceptible individuals within a network.

Basically, in many studies that feature the vaccination game, it is often assumed that every individual behaves according to the same perceived risks of infection. In reality, it is very possible that some individuals within a population overestimate both the incidence of a disease and the perceived risks of infection and those oversensitive ones can consistently take vaccines. Based on this point, Liu et al. (2012) investigated the impact of the presence of stubborn (i.e., committed) vaccinated individuals who always hold a vaccination strategy, with regard to vaccination coverage and epidemiological dynamics. They found that a small fraction of these stubborn individuals who always take vaccines can inhibit the formation of clusters of susceptible individuals; these stubborn individuals thus help to promote vaccination coverage by acting as 'steadfast role models' in the population.

However, it is not necessarily so that stubborn individual behaviour always features the taking of vaccines. Some people always underestimate the perceived risks of infection, due to a lack of knowledge about infectious disease - and/or they may overestimate the perceived risks of vaccination based on scientifically groundless information. As Jansen et al. (2003) pointed out, it is actually a serious cause of a reduction in vaccination coverage - that is, separate and aside from the stubborn vaccinated individuals, there also exist within the population stubborn unvaccinated individuals who always take a no-vaccination strategy. Accordingly, within a population, stubborn individuals who always hold their own strategy tend to fall into one of two types: stubborn vaccinated and unvaccinated individuals.

For the above reasons, in this study, we look to examine how the presence of stubborn vaccinated and unvaccinated individuals (i.e., 'zealots') affects individual-level vaccination behaviour and the spread of infectious disease within a social network and under a voluntary vaccination policy. To this end, we integrate an evolutionary game theoretic approach with simple epidemiological dynamics - namely, susceptible-infection-recovered (SIR) dynamics (Kermack and McKendrick, 1927). We focus on a flu-like infection as a typical seasonal (periodic) infectious disease, because the protective efficacy against such infectious diseases usually lasts for one season only, and so individuals need to decide every season whether or not to be vaccinated.

This paper is organised as follows. The details of our combined model with epidemiological dynamics and a decision-making process with regard to vaccination (vaccination game), as well as methods of computational simulation, are described in Section 2. Next, the results obtained for the respective networks are presented in Section 3, along with relevant discussion. Then, conclusions and suggestions for future research are presented in Section 4. Finally, complementary results with regard to Sections 2 and 3 are shown as the Supplementary material. 


\section{Model and simulation methods}

\subsection{Vaccination game}

We consider a population in which every member undertakes vaccination behaviour adaptively in the midst of a seasonal and periodic flu-like disease. Stubborn vaccinated individuals (SVs) and stubborn unvaccinated individuals (SUs) are present in the population. Immunisation efficacy is usually impermanent against such infectious disease, and so individuals need to take a vaccine each season to acquire immunity against the infectious disease of that season. As in $\mathrm{Fu}$ et al. (2011), the vaccination dynamics of our model consist of two stages.

In the first stage, the vaccination campaign, each individual decides in advance of the seasonal epidemic whether or not take a vaccine, as every individual is exposed to the risk of becoming infected. An individual who chooses a strategy involving the taking of the vaccine (including SVs) incurs the cost of vaccination $C_{\mathrm{v}}$, which may include the monetary cost and any perceived risks, such as complications and adverse side effects (Galvani et al., 2007). For the sake of simplicity, we assume that a (stubborn) vaccinated individual acquires perfect immunity against the disease during the season; on the other hand, (stubborn) unvaccinated individuals are exposed to the risk of becoming infected that same season. At the second stage, the epidemic season, randomly selected susceptible individuals (including SUs) $I_{0}$ who take the no-vaccination strategy are identified as those initially infected by the epidemic strain; the disease then transmits in the population via a social network, in line with SIR dynamics. In the SIR model, a population is divided into three groups: susceptible, infectious, and recovered ones. Susceptible individuals become infected, with the parameter $\beta$-the disease transmission rate per day per person by coming into contact with infectious ones; they themselves then become infectious. They recover from their disease, with the parameter $\gamma-$ the recovery rate per day (i.e., the inverse of the mean number of days required to recover from one's own disease). Here, recovered individuals, as well as (stubborn) vaccinated ones, have acquired perfect immunity against the infectious disease. In numerically simulating the SIR dynamics in a social network, we apply the Gillespie (1977) algorithm. For details on how to apply the Gillespie algorithm to this epidemiological process, see Fu et al. (2011).

An epidemic season is terminated by the disappearance of every infectious individual from the population. Infected individuals (including infected SUs) during the epidemic season incur the cost of infection $C_{\mathrm{i}}$, which may include the cost of healthcare, lost productivity, the possibility of pain or mortality, and so on (Galvani et al., 2007). In contrast, successful individuals (including healthy SUs) who do not get the vaccine and yet remain healthy are called free riders, and they avoid any payment. For simplicity, we rescale the cost without loss of generality by defining the relative cost of vaccination $C_{\mathrm{r}}=C_{\mathrm{v}} / C_{\mathrm{i}}$ (For a typical seasonal flu, an estimation shows that the value of $C_{\mathrm{r}}$ may be not so large in real world (Fu et al., 2011; Galvani et al., 2007). However, any estimation has many assumptions and there must be fluctuation and unknowability in some factors contributing to the final cost, so that we cannot give an upper bound of $C_{\mathrm{r}}$ (obviously, it must be smaller than one). Thus, we here set $0 \leq C_{\mathrm{r}} \leq 1$ to take every possibility into 
consideration). Then, according to the final epidemic state (vaccination strategy and health condition) following the epidemic season, a payoff to an individual $i, \pi_{i}$, is stipulated and takes one of three types:

$$
\pi_{i}= \begin{cases}-C_{\mathrm{v}}, & \text { vaccination and healthy; } \\ -1, & \text { no-vaccination and infected; } \\ 0, & \text { no-vaccination and healthy. }\end{cases}
$$

\subsection{Strategy adaptation}

At the end of every two stages, each individual re-examines whether to change or retain her own vaccination strategy, via an imitation process that is based on the payoffs of her and her neighbours for the next season; this process excludes stubborn individuals, as they will retain their own strategies regardless of these factors (Liu et al., 2012). The details of the rules for strategy adaptation are as follows. An individual $i$ randomly choose a neighbour individual $j$ in the network. Let $s_{i}$ and $s_{j}$ denote the strategies (vaccination or no-vaccination) of individual $i$ and her neighbour $j$, respectively. The probability $P\left(s_{i} \leftarrow s_{j}\right)$ - where the individual $i$ who takes the strategy $s_{i}$ imitates the strategy $s_{j}$ of individual $j$ - is given by a pairwise comparison of their payoff difference $\pi_{i}$ $-\pi_{j}$, according to the Fermi function (Traulsen et al., 2007; Szabo and Toke, 1998),

$$
P\left(s_{i} \leftarrow s_{j}\right)=\frac{1}{1+\exp \left[\left(\pi_{i}-\pi_{j}\right) / \kappa\right]},
$$

where $\kappa$ denotes the selection pressure (the sensitivity of individuals to the difference in the payoffs). For $\kappa \rightarrow \infty$ (weak selection pressure, not information completeness about the payoffs of individuals), the probability $P\left(s_{i} \leftarrow s_{j}\right)$ approaches $1 / 2$ asymptotically, regardless of their payoff difference $\pi_{i}-\pi_{j}$, because an individual $i$ is ultimately insensitive to the payoff difference against another individual $j$. On the other hand, for $\kappa$ $\rightarrow 0$ (strong selection pressure), they definitely update the successful strategy that earns the higher payoff, even if the payoff difference is very small, because they are ultimately sensitive to the payoff difference. This comparison rule has been widely accepted in evolutionary game theory (Wang et al., 2013). In this study, we set $\kappa=1$, without loss of generality. The value of $\kappa$ reflects behaviour wherein individuals basically adopt a successful strategy; however, they occasionally do fail, because of irrationality or the mistakes made by ordinary individuals. Note that, if an individual $i$ and her neighbour $j$ were to take the same strategy, and/or if an individual $i$ were a stubborn individual (SV or SU), then $P\left(s_{i} \leftarrow s_{j}\right)=0$ - in other words, she will hold her current strategy, regardless of the payoff difference. Figure 1 shows the flow of our vaccination dynamics model described thus far. 
Figure 1 Flow of our model in which vaccination dynamics are modelled as a two-stage process

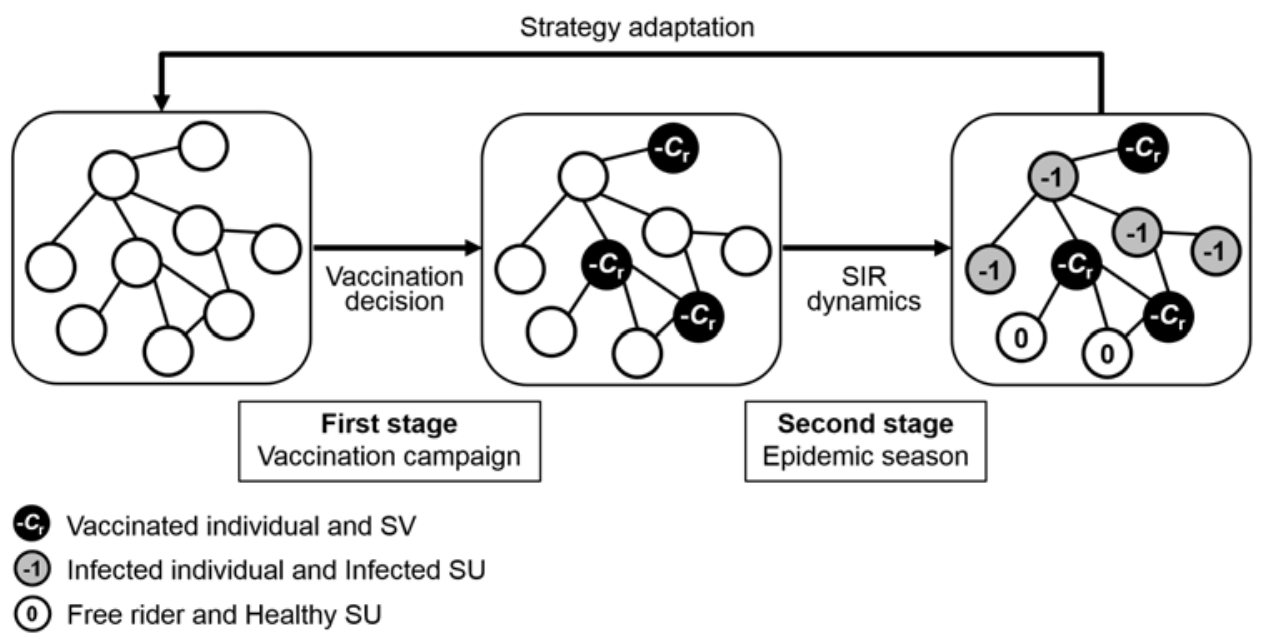

\subsection{Simulation setting}

In our simulations, during the initial stage, half the population of vaccinated individuals (including a certain fraction of SVs, $f_{\mathrm{SV}}$ ) and half the population of the unvaccinated (susceptible) individuals (including a certain fraction of SUs, $f_{\mathrm{SU}}$ ) are randomly distributed over the population; the population size is 4,900 . We assume that the fractions of stubborn individuals are not larger than that of normal individuals - that is, $f_{\mathrm{SV}}+f_{\mathrm{SU}} \leq$ 0.5. Both types of stubborn individuals (i.e., SVs and SUs) hold the same state throughout a simulation - in other words; they occupy the same nodes, while holding the same strategy from the first season to the final season. After that, an epidemic strain randomly infects susceptible individuals, as the initial number $I_{0}=5$; the epidemic then spreads according to SIR dynamics throughout a social network. It is widely known that the features of disease propagation significantly depend on the network structure involved (Keeling and Eames, 2005). In addition, the network structure of flu propagation in human society has been yet identified explicitly, even though mathematical modelling studies have been advanced in sexually transmitted diseases (Colgate et al., 1989; Liljeros et al., 2001; Schneeberger et al., 2004) and flu infections in animals such as avian flu (Small et al., 2007). Thus, in this study, we consider a square lattice with a von Neumann neighbourhood and a Barabási-Albert scale-free (BA-SF) network with an average degree $\langle k\rangle=4$ (Barabási and Albert, 1999) as a typical population structure; we then calibrate the value of $\beta$, such that the final proportion of infected individuals across each network will be 0.9 (see Supplementary material) (Fu et al., 2011). Here, we set $\beta=0.46$ for the square lattice and $\beta=0.55$ for the BA-SF network, and $\gamma=1 / 3$ as the recovery rate. A typical seasonal flu is assumed to determine these disease parameters. The vaccination coverage and final epidemic size are updated by iterating each two-stage process (the vaccination campaign and the epidemic season) and the imitation process of the vaccination strategy. The equilibrium (stable-state) results shown in Figures 2 (except for snapshots) to 7 represent average fractions over the final 1,000 of 3,000 iterations in 100 independent simulations. 


\section{Results and discussion}

\subsection{General discussion}

Each of (A)-(D) in Figures 2 and 5 show stable-state values for vaccination coverage and final epidemic size in each network as functions of the relative cost of vaccination $C_{\mathrm{r}}$, for different fractions of SUs $\left(f_{\mathrm{SU}}\right)$ when the fraction of SVs $\left(f_{\mathrm{SV}}\right)$ equals 0 or 0.1 . The cases shown in the solid lines $\left(f_{\mathrm{SV}}=f_{\mathrm{SU}}=0\right)$ in these figures - where there is neither an SV nor an SU in the population - correspond to the results in Fu et al. (2011). The cases shown in the dashed lines $\left(f_{\mathrm{SU}}=0\right)$, where there is no $\mathrm{SU}$ in the population, correspond to the results in Liu et al. (2012). The larger the $f_{\mathrm{Su}}$ becomes, the more vaccination coverage declines - and, as a result, the more the final epidemic size increases with the same values of $C_{\mathrm{r}}$ over the full range of $C_{\mathrm{r}}$.

Figures 3 and 6 show vaccination coverage and final epidemic size. Figures 4 and 7 comprise another set of contour diagrams that show the difference in the vaccination coverage and final epidemic size relative to the case where $f_{\mathrm{SV}}=f_{\mathrm{SU}}=0$ is assumed (plotted at the origin in Figures 3 and 6), as functions of the fraction of SVs $\left(f_{\mathrm{sV}}\right)$ and that of SUs $\left(f_{\mathrm{su}}\right)$,for different $C_{\mathrm{r}}$ in each network. As can be seen from these counter diagrams (in particular, Figures 4 and 7), the presence of SUs exerts an adverse effect on the spread of infectious disease, in comparison to the case where $f_{\mathrm{SV}}=f_{\mathrm{SU}}=0$, for the range where $C_{\mathrm{r}}$ is small - which corresponds to the negative region (less vaccination than the case of $f_{\mathrm{SV}}=f_{\mathrm{SU}}=0$ ) in Figure 4 and the positive region (larger final epidemic size than the case of $f_{\mathrm{SV}}=f_{\mathrm{SU}}=0$ ) in Figure 7. On the other hand, as $C_{\mathrm{r}}$ becomes larger, the presence of SVs exerts a favourable effect in preventing the spread of disease, and this corresponds to the positive and negative regions in Figures 4 and 7, respectively. This tendency is attributable to each individual's vaccination incentive. For the range where $C_{\mathrm{r}}$ is small, the vaccination incentive is high in the first place, because an individual can take a vaccine at a low cost; hence, the favourable effect of the presence of SVs in promoting vaccination behaviour is weak. Meanwhile, if $C_{\mathrm{r}}$ is large, the vaccination incentive is already low (since the vaccination cost is expensive), and so in this case, the adverse effect produced by the presence of SUs in promoting no-vaccination behaviour is weak.

The effect of the presence of SUs in the case of a BA-SF network is much more marked than that of a lattice population case. This is because the degree heterogeneity of the BA-SF network makes it easier for disease to propagate than in a lattice population - one of archetype homogeneous graphs (see Figure 8) (Keeling and Eames, 2005). In such a network, individuals who have many neighbours (so-called hub individuals) tend to take vaccines, since they can easily become infected [Figures 5(E) and (F)]. Moreover, the vaccination/no-vaccination behaviour of many individuals who have relatively few neighbours is promoted by imitating the strategy of hub individuals (Fu et al., 2011). Therefore, the adverse effect on disease propagation that is produced by the presence of SUs is notably enhanced in the population of the BA-SF network - and more so than in lattice populations - since it is intrinsically difficult to promote voluntary vaccination behaviour in lattice populations.

Concerning the lower panels of Figures 4 and 7 (final epidemic size), we should note the following. From the social application viewpoint, it might be important to determine whether or not the region above the 45-degree dashed line that is still marked with a negative value (i.e., smaller final epidemic size than the case of $f_{\mathrm{SV}}=f_{\mathrm{SU}}=0$ ) does exist, and if so, how wide that region is. In this particular region, disease propagation is more 
efficiently suppressed than is the case with $f_{\mathrm{SV}}=f_{\mathrm{SU}}=0$, even though the number of SUs is larger than that of vaccinated individuals. In this sense, obviously, the lattice population more effectively assists in preventing disease propagation than the BA-SF network, since the aforementioned region in the lattice population case appears even in a smaller- $C_{\mathrm{r}}$ setting.

To examine the details pertaining to the effect of population structure, the results we obtained, as well as relevant discussion of the respective networks, are offered below in the following sections.

\subsection{Lattice populations}

Figures 2(E) and (F) illustrate typical snapshots of the system after it approaches equilibrium for $f_{\mathrm{SU}}=0$ and 0.2 , when $C_{\mathrm{r}}=0.2$ and $f_{\mathrm{SV}}=0$. For the $f_{\mathrm{SU}}=0$ in Figure 2(E), the vaccinated individuals form some small clusters that represent about $17 \%$ of the population, and about $27 \%$ of the population gets infected. For $f_{\mathrm{SU}}=0.2$ in Figure 2(F), about $9 \%$ of the vaccinated individuals form some small clusters, and about $62 \%$ of the population gets infected. Therefore, the presence of SUs slightly promotes no-vaccination behaviour in the population, and a large epidemic ensues. The reason for this is explained as follows. If SUs are present in the population, they provoke neighbouring individuals to imitate the SUs' strategy, which ultimately leads to reduced vaccination coverage. As a result, larger clusters of susceptible individuals form, and they comprise a larger final proportion of infected individuals. However, if vaccination coverage were to decline further, it would be difficult for individuals to take a free ride on the benefit - that is, the unvaccinated individuals cannot be protected indirectly by the vaccinated individuals. Therefore, the SUs cannot 'seduce' individuals not to voluntarily get vaccinated, and a further reduction of vaccination coverage will not occur.

Figures $2(\mathrm{G})$ and $(\mathrm{H})$ illustrate typical snapshots of the system after the system approaches equilibrium for $f_{\mathrm{SU}}=0$ and 0.2 , when $C_{\mathrm{r}}=0.2$ and $f_{\mathrm{SV}}=0.1$. For $f_{\mathrm{SU}}=0$ in Figure $2(\mathrm{G})$, about $63 \%$ of the vaccinated individuals, as a proportion of the total population, form large clusters that are evenly distributed throughout the lattice populations (Liu et al., 2012); about $1 \%$ of the total population becomes infected. For the case where $f_{\mathrm{SU}}=0.2$ in Figure $2(\mathrm{H}), 27 \%$ of the vaccinated individuals form small clusters that are evenly distributed throughout the lattice populations; about $5 \%$ become infected, even when $f_{\mathrm{SV}}<f_{\mathrm{SU}}$. As can be seen from these figures - specifically, Figure $2(\mathrm{H})$ - the SVs who are distributed randomly throughout the lattice populations promote individual vaccination behaviour, and help to form clusters of vaccinated individuals who are evenly distributed throughout the population, even when $f_{\mathrm{SV}}<f_{\mathrm{SU}}$; its impact inhibits SUs from 'seducing' the entire population to avoid vaccination and, as a result, the epidemic is greatly mitigated. The detailed reason for this is as follows. As described above, to prevent an infectious disease from spreading in the lattice populations, it is effective to inhibit susceptible individuals from forming clusters (Ndeffo Mbah et al., 2012). The promotion of vaccination coverage is effective in doing this; it is also effective to distribute the clusters of the vaccinated individuals evenly throughout the network, because any of these clusters shuts out the initial infected ones who can appear anywhere in the network. For the case where $f_{\mathrm{SV}}=f_{\mathrm{SU}}=0$ in Figure 2(E), infectious disease spreads widely in the population, because the clusters of vaccinated individuals are small - and thus, the vaccination coverage is low. Moreover, they do not 
distribute evenly throughout the network. In contrast, in the case where $f_{\mathrm{SV}}=0.1$, $f_{\mathrm{SU}}=0.2$ in Figure $2(\mathrm{H})$, not only does it promote imitation behaviour with respect to vaccination, but it also helps form and evenly distribute clusters of vaccinated individuals - even though the cluster sizes are small - and thus preclude the clustering of susceptible individuals, thanks to the presence of SVs who are randomly distributed throughout the population. Therefore, the small fraction of SVs has a large impact on the suppression of disease propagation, thanks to the imitation process and the local interactions of structured populations [for a detailed discussion, see Liu et al. (2012)].

Figure 2 Vaccination coverage (A and $\mathrm{C}$ ) and final epidemic size (B and D) as functions of the relative cost of vaccination $C_{\mathrm{r}}$ for different fractions of SUs $\left(f_{\mathrm{SU}}\right)$ when the fraction of SVs $\left(f_{\mathrm{SV}}\right)$ equals $0(\mathrm{~A}$ and $\mathrm{B})$ and $0.1(\mathrm{C}$ and $\mathrm{D})$; and typical snapshots of systems in the equilibrium state when $C_{\mathrm{r}}=0.2(\mathrm{E}-\mathrm{H})$ in the lattice populations

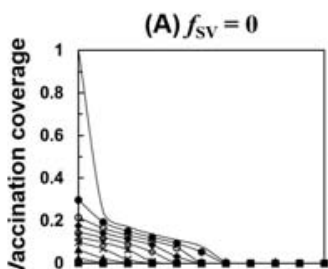

(C) $f_{\mathrm{sv}}=0.1$

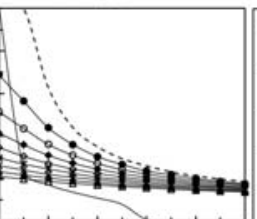

(B) $f_{\mathrm{Sv}}=0$

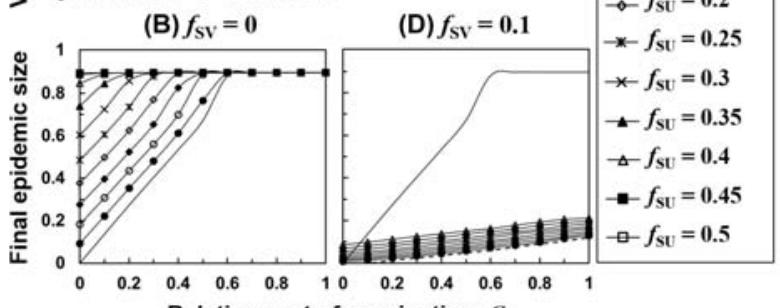

Relative cost of vaccination, $C_{r}$
(E) $f_{\mathrm{SV}}=f_{\mathrm{SU}}=0$

(G) $f_{\mathrm{Sv}}=0.1, f_{\mathrm{SU}}=0$
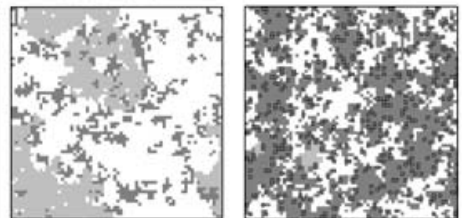

(F) $f_{\mathrm{SV}}=0, f_{\mathrm{SU}}=0.2(\mathrm{H}) f_{\mathrm{SV}}=0.1, f_{\mathrm{SU}}=0.2$
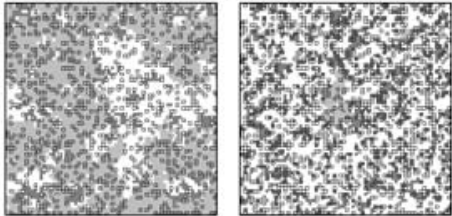

Vaccinated individual $\square$ SV

Infected individual

$\square$ Infected SU

$\square$ Healthy su

Figure 3 Contour diagrams of vaccination coverage (upper six panels) and final epidemic size (lower six panels), as functions of the fraction of SVs $\left(f_{\mathrm{SV}}\right)$ and of SUs $\left(f_{\mathrm{SU}}\right)$ when $C_{\mathrm{r}}=0$ $(\mathrm{A}$ and $\mathrm{B}), 0.1(\mathrm{C}$ and $\mathrm{D}), 0.2(\mathrm{E}$ and $\mathrm{F}), 0.5(\mathrm{G}$ and $\mathrm{H}), 0.6(\mathrm{I}$ and $\mathrm{J})$, and $1(\mathrm{~K}$ and $\mathrm{L})$ in the lattice populations
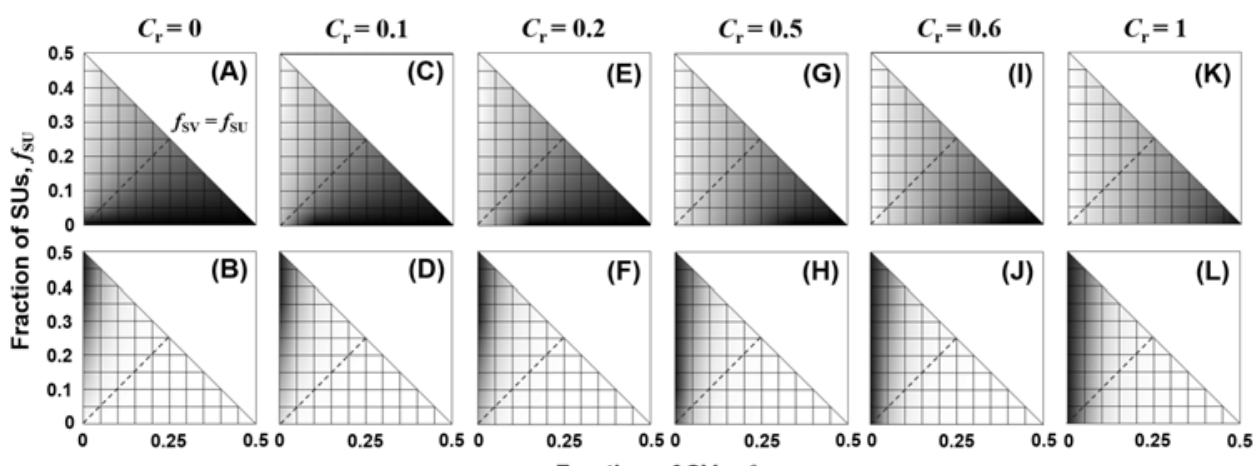

Fraction of SVs, $f_{s v}$

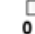


Figure 4 Contour diagrams of differences in vaccination coverage (upper six panels) and final epidemic size (lower six panels) between the case at origin and the others when $C_{\mathrm{r}}=0$ (A and B), $0.1(\mathrm{C}$ and $\mathrm{D}), 0.2$ (E and F), $0.5(\mathrm{G}$ and $\mathrm{H}), 0.6$ (I and $\mathrm{J})$, and 1 (K and $\mathrm{L}$ ) in the lattice populations
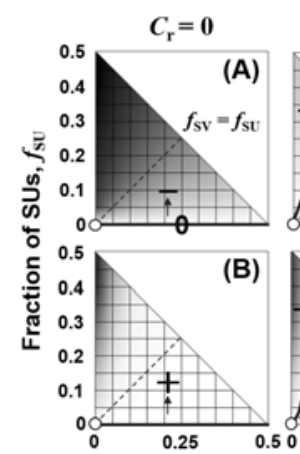
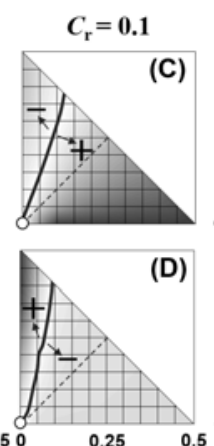
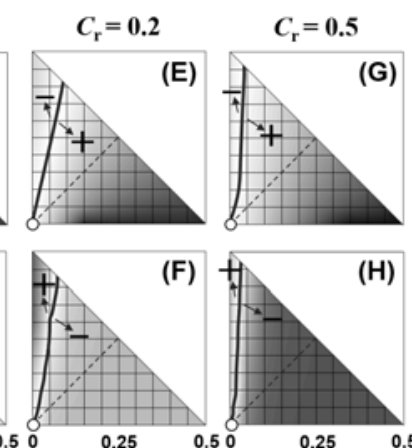

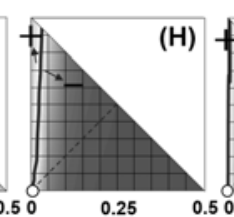

(H)

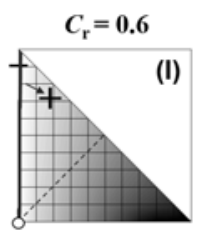

(J)

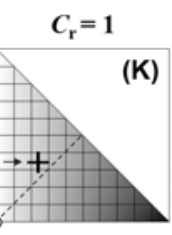

(L)

Fraction of SVs, $f_{\mathrm{sv}}$

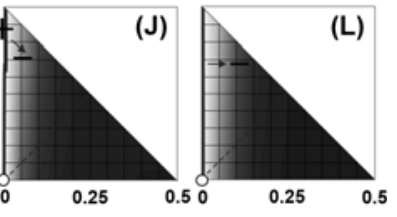

\subsection{Barabási-Albert scale-free networks}

Figure 5(E) shows the fraction of vaccinated individuals as a function of the number of neighbours when $C_{\mathrm{r}}=0.2$ and $f_{\mathrm{SV}}=0$. As seen in this figure, there is a general tendency wherein the greater the number of neighbours (with a higher degree) that individuals have, the more of them that tend to be vaccinated, regardless of the presence of SUs. However, in the case where $f_{\mathrm{SU}}=0.2$, not only is it that the vaccination coverage in the whole population is more inhibited than in the case of $f_{\mathrm{SV}}=f_{\mathrm{SU}}=0$, but also the fraction of vaccinated individual individuals among the hub individuals is more scattered than is the case with $f_{\mathrm{SV}}=f_{\mathrm{SU}}=0$. This is attributed to the fact that SUs are randomly chosen in the population. It is well known that the BA-SF network is one type of scale-free network in which, according to power law, a great majority of individuals have few connections and there are relatively few hubs. It is important to note that in a BA-SF network, the hubs who can become 'super-spreaders' should be sure to take vaccines, in order to suppress disease propagation (Keeling and Eames, 2005). Thus, even if a single hub is selected as a SU, this could easily lead to a large reduction in vaccination coverage among individuals of the same degree within that particular hub. Because of this reduction in vaccination coverage among hubs, those who have fewer contacts connected to the hubs tend to imitate the no-vaccination strategy. These facts yield the result shown in Figure 5(E).

Figure $5(\mathrm{~F})$ shows the fraction of vaccinated individuals as a function of the number of neighbours when $C_{\mathrm{r}}=0.2$ and $f_{\mathrm{SV}}=0.1$. As is almost seen in Figure $5(\mathrm{E})$, for the case where $f_{\mathrm{SU}}=0.2$, the fraction of vaccinated individuals across the full range of degrees largely declines, due to the presence of SUs - even though SVs are present in the population. As mentioned, no-vaccination behaviour among hubs brings about a large reduction in vaccination coverage - and, consequently, great disease propagation when they are selected as SUs. Therefore, the adverse effect of the presence of SUs outpaces the favourable effect of SVs, particularly for the range where $C_{\mathrm{r}}$ is small. 
Figure 5 Vaccination coverage (A and C) and final epidemic size (B and D) as functions of the relative cost of vaccination $C_{\mathrm{r}}$ for different fractions of SUs $\left(f_{\mathrm{SU}}\right)$ when the fraction of SVs $\left(f_{\mathrm{SV}}\right)$ equals $0(\mathrm{~A}$ and $\mathrm{B})$ and $0.1(\mathrm{C}$ and $\mathrm{D})$; and the fraction of vaccinated individuals as a function of the number of neighbours when $C_{\mathrm{r}}=0.2(\mathrm{E}$ and $\mathrm{F})$ in the BA-SF network

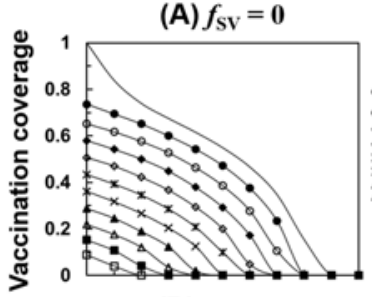

(B) $f_{\mathrm{SV}}=0$

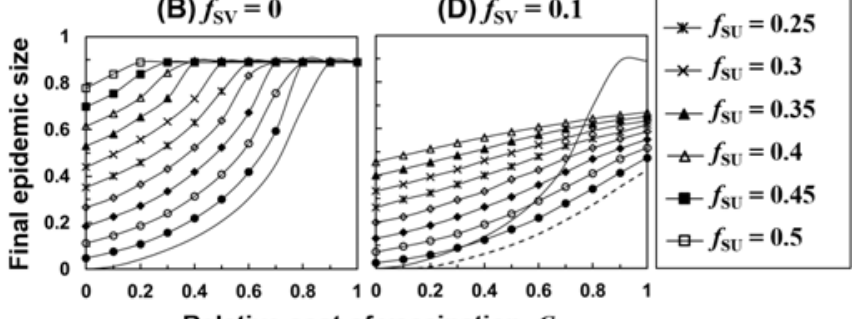

Relative cost of vaccination, $C_{\mathrm{r}}$

(C) $f_{\mathrm{sv}}=0.1$

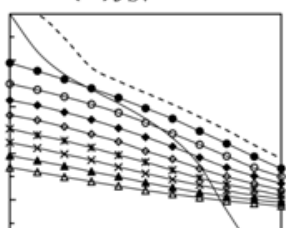

$-f_{\mathrm{SV}}=f_{\mathrm{SU}}=0$

-- $f_{\mathrm{SU}}=0$

- $f_{\mathrm{SU}}=0.05$

$-0-f_{\mathrm{SU}}=0.1$

- $f_{\mathrm{SU}}=0.15$

$\rightarrow f_{\mathrm{sv}}=0.2$

$\leftarrow f_{\mathrm{SU}}=0.3$

$\triangle f_{\mathrm{sU}}=0.4$

$$
\text { . }
$$

Figure 6 Contour diagrams of vaccination coverage (upper six panels) and final epidemic size (lower six panels) as functions of the fraction of SVs $\left(f_{\mathrm{SV}}\right)$ and of SUs $\left(f_{\mathrm{SU}}\right)$ when $C_{\mathrm{r}}=0$ (A and B), 0.1 (C and D), 0.5 (E and F), $0.8(\mathrm{G}$ and H), 0.9 (I and J), and $1(\mathrm{~K}$ and $\mathrm{L}$ ) in the BA-SF network

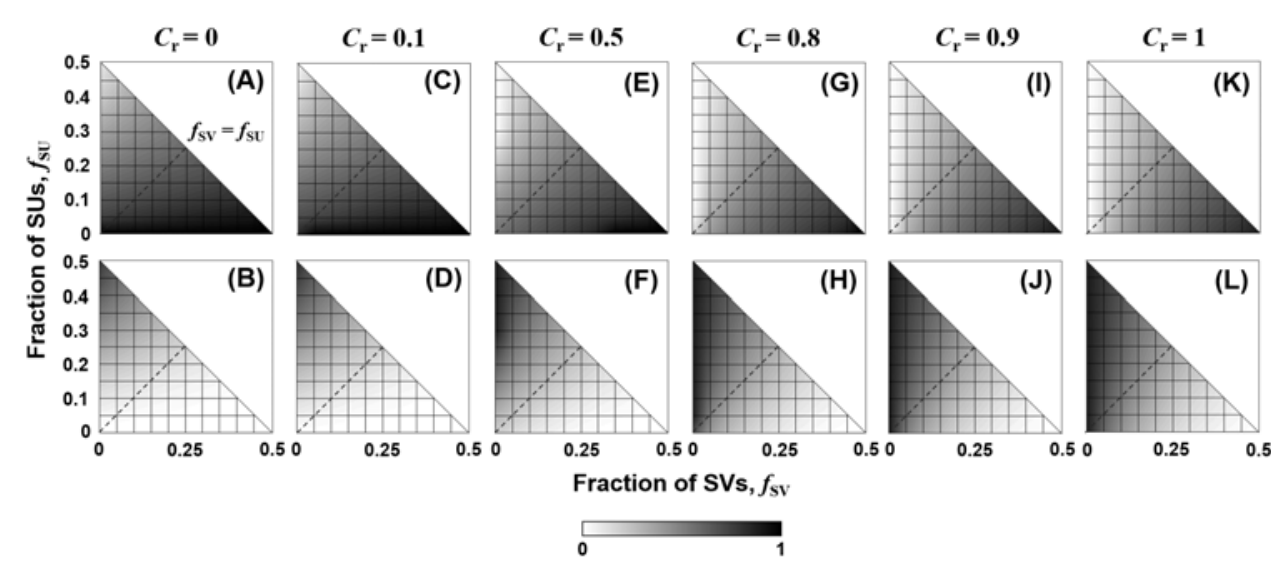


Figure 7 Contour diagrams of differences in vaccination coverage (upper six panels) and final epidemic size (lower six panels) between the case at origin and the others when $C_{\mathrm{r}}=0$ (A and B), $0.1(\mathrm{C}$ and $\mathrm{D}), 0.5(\mathrm{E}$ and $\mathrm{F}), 0.8(\mathrm{G}$ and $\mathrm{H}), 0.9$ (I and $\mathrm{J})$, and $1(\mathrm{~K}$ and $\mathrm{L})$ in the BA-SF network

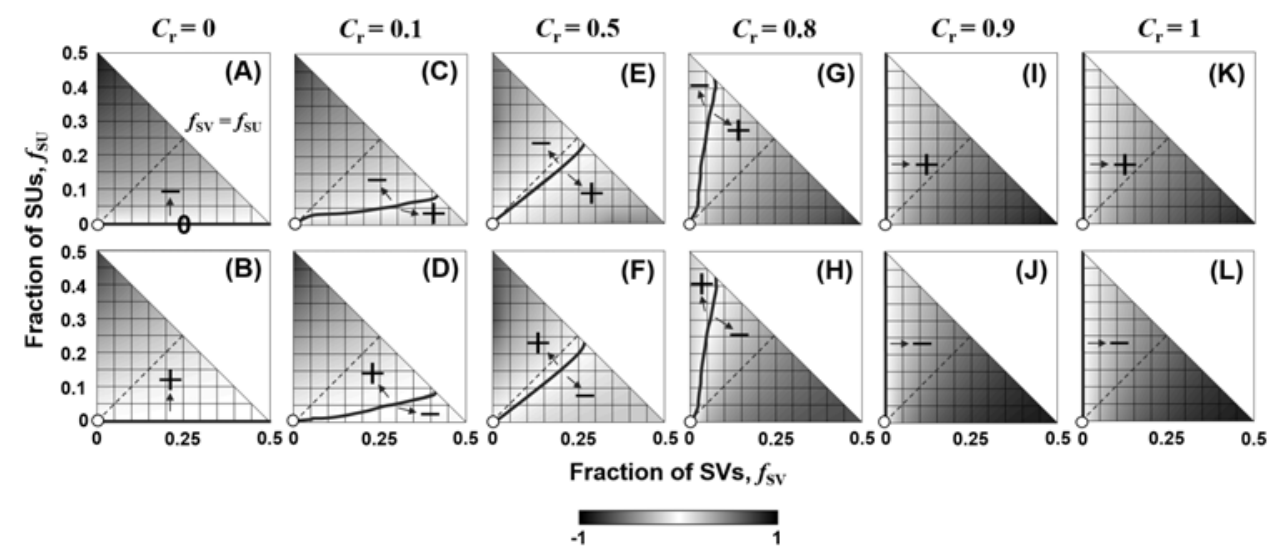

\section{Conclusions}

In this study, we investigated how the presence of stubborn vaccinated or unvaccinated individuals who are intransigent about their own vaccination strategy can affects other individuals' decision-making vis-à-vis whether or not to get a vaccination themselves, as well as the spread of flu-like infectious disease. Consequently, we found that the influence of the presence of both types of stubborn individuals greatly depends on their proportion within the population, as well as the population structure and the relative cost of vaccination $C_{\mathrm{r}}$.

Lattice populations can greatly prevent disease propagation by distributing SVs evenly across the population at a wider range of $C_{\mathrm{r}}$, even though the fraction of SUs is greater than that of SVs. In contrast, for the BA-SF network, disease propagation cannot be prevented, since the hub individuals may be selected as SUs when the $C_{\mathrm{r}}$ has a lower-than-moderate value - even when the SVs are present in the population. Based on our results, it is suggested that we pay attention not only to the regional network structures and the cost of vaccination, but also to the presence of SUs when we examine intervenient methods by which to prevent disease propagation.

In our simulations, we assumed that individuals are situated within a social network in the midst of a flu-like disease. However, the epidemic parameters - such as the transmission rate, $\beta$ - have a profound effect on disease propagation (Keeling and Eames, 2005). Besides, for simplicity, we assumed that individuals acquire perfect immunity from taking a vaccine against a seasonal infectious disease during an epidemic season - that is, vaccinated individuals definitely will not become infected during the epidemic season. In reality, however, for some infectious diseases the immunisation efficacy and effectiveness of a vaccine are not always perfect (Hoffman, 1996; Sudfeld et al., 2010). In future work, we must investigate the impact of the presence of stubborn individuals under various epidemic-parameter conditions; additionally, vaccination 
efficacy and effectiveness must be considered, and doing so would allow us to make more realistic proposals for preventing epidemics.

\section{References}

Anderson, R.M. and May, R.M. (1991) Infectious Diseases of Humans: Dynamics and Control, Oxford University Press, New York.

Bailey, N.T.J. (1975) The Mathematical Theory of Infectious Diseases and Its Applications, 2nd ed., Griffin, London.

Barabási, A.L. and Albert, R. (1999) 'Emergence of scaling in random networks', Science, Vol. 286, No. 5439, pp.509-512.

Basu, S., Chapman, G.B., and Galvani, A.P. (2008) 'Integrating epidemiology, psychology, and economics to achieve HPV vaccination targets', Proceedings of the National Academy of Sciences of the United States of America, Vol. 105, No. 48, pp.19018-19023.

Bauch, C.T. (2005) 'Imitation dynamics predict vaccinating behaviour', Proceedings of the Royal Society B, Vol. 272, No. 1573, pp.1669-1675.

Bauch, C.T. and Bhattacharyya, S. (2012) 'Evolutionary game theory and social learning can determine how vaccine scares unfold', PLoS Computational Biology, Vol. 8, No, 4, pp.e1002452.

Bauch, C.T. and Earn, D.J.D. (2004) 'Vaccination and the theory of games', Proceedings of the National Academy of Sciences of the United States of America, Vol. 101, No. 36, pp.13391-13394.

Bauch, C.T., Anonychuk, A.M., Pham, B.Z., Gilca, V., Duval, B. and Krahn, M.D. (2007) 'Cost-utility of universal hepatitis a vaccination in Canada', Vaccine, Vol. 25, No. 51, pp.8536-8548.

Bauch, C.T., Galvani, A.P. and Earn, D.J.D. (2003) 'Group interest versus self-interest in smallpox vaccination policy', Proceedings of the National Academy of Sciences of the United States of America, Vol. 100, No. 18, pp.10564-10567.

Brisson, M. and Edmunds, W. (2003) 'Economic evaluation of vaccination programs: the impact of herd-immunity', Medical Decision Making, Vol. 23, No. 1, pp.76-82.

Chapman, G.B. and Coups, E.J. (1999) 'Predictors of influenza vaccine acceptance among healthy adults', Preventive Medicine, Vol. 29, No. 4, pp.249-262.

Chapman, G.B. and Coups, E.J. (2006) 'Emotions and preventive health behavior: worry, regret, and influenza vaccination', Health Psychology, Vol. 25, No. 1, pp.82-90.

Colgate, S.A., Stanley, E.A., Hyman, J.M., Layne, S.P. and Qualls, C. (1989) 'Risk behavior-based model of the cubic growth of acquired immunodeficiency syndrome in the United States', Proceedings of the National Academy of Sciences of the United States of America, Vol. 86, No. 12, pp.4793-4797.

Fu, F., Rosenbloom, D.I., Wang, L. and Nowak, M.A. (2011) 'Imitation dynamics of vaccination behaviour on social networks', Proceedings of the Royal Society B, Vol. 278, No. 1702, pp.42-49.

Fukuda, E., Kokubo, S., Tanimoto, J., Wang, Z., Hagishima, A. and Ikegaya, N. (2014) 'Risk assessment for infectious disease and its impact on voluntary vaccination behavior in social networks', Chaos, Solitons \& Fractals, Vol. 68, pp.1-9, ISSN: 0960-0779.

Funk, S., Salathé, M. and Jansen, V.A.A. (2010) 'Modelling the influence of human behaviour on the spread of infectious diseases: a review', Journal of the Royal Society Interface, Vol. 7, No. 50, pp.1247-1256.

Galvani, A.P., Reluga, T.C. and Chapman, G.B. (2007) 'Long-standing influenza vaccination policy is in accord with individual self-interest but not with the utilitarian optimum', Proceedings of the National Academy of Sciences of the United States of America, Vol. 104, No. 13, pp.5692-5697. 
Gillespie, D.T. (1977) 'Exact stochastic simulation of coupled chemical reactions', The Journal of Physical Chemistry, Vol. 81, No. 25, pp.2340-2361.

Hoffman, S.L. (Ed.) (1996) Malaria Vaccine Development: a Multi-Immune Response Approach, American Society of Microbiology, Washington, DC.

Jansen, V.A., Stollenwerk, N., Jensen, H.J., Ramsay, M.E., Edmunds, W.J., and Rhodes, C.J. (2003) 'Measles outbreaks in a population with declining vaccine uptake', Science, Vol. 301, No. 5634 , p.804.

Keeling, M.J. and Eames, K.T.D. (2005) 'Networks and epidemic models', Journal of the Royal Society Interface, Vol. 2, No. 4, pp.295-307.

Kermack, W.O. and McKendrick, A.G. (1927) 'A contribution to the mathematical theory of epidemics', Proceedings of the Royal Society of London A, Vol. 115, No. 772, pp.700-721.

Liljeros, F., Edling, C.R., Amaral, L.A.N., Stanley, H.E. and Åberg, Y. (2001) 'The web of human sexual contacts', Nature, Vol. 411, No. 6840, pp.907-908.

Liu, X.T., Wu, Z.X. and Zhang, L. (2012) 'Impact of committed individuals on vaccination behavior', Physical Review E, Vol. 86, No. 5, p.051132.

Meyers, A.L., Pourbohloul, B., Newman, M.E.J., Skowronski, D.M. and Brunham, R.C. (2005) 'Network theory and SARS: predicting outbreak diversity', Journal of Theoretical Biology, Vol. 232, No. 1, pp.71-81.

Ndeffo Mbah, M.L., Liu, J., Bauch, C.T., Tekel, Y.I., Medlock, J., Meyers, L.A. and Galvani, A.P. (2012) 'The impact of imitation on vaccination behavior in social contact networks', PLoS Computational Biology, Vol. 8, No. 4, p.e1002469.

Pastor-Satorras, R. and Vespignani, A. (2001) 'Epidemic spreading in scale-free networks', Physical Review Letters, Vol. 86, No. 14, pp.3200-3203.

Perisic, A. and Bauch, C.T. (2009) 'Social contact networks and disease eradicability under voluntary vaccination', PLoS Computational Biology, Vol. 5, No. 2, p.e1000280.

Schimit, P. and Monteiro, L.A. (2011) 'A vaccination game based on public health actions and personal decisions', Ecological Modelling, Vol. 222, No. 9, pp.1651-1655.

Schneeberger, A., Mercer, C.H., Gregson, S.A.J., Ferguson, N.M., Nyamukapa, C.A., Anderson, R.M., Johnson, A.M. and Garnett, G.P. (2004) 'Scale-free networks and sexually transmitted diseases: a description of observed patterns of sexual contacts in Britain and Zimbabwe', Sexually Transmitted Diseases, Vol. 31, No. 6, pp.380-387.

Small, M., Walker, D.M. and Tse, C.K. (2007) 'Scale-free distribution of avian influenza outbreaks', Physical Review Letters, Vol. 99, No. 18, pp.188702-188705.

Sudfeld, C.R., Navar, A.M. and Haksey, N.A. (2010) 'Effectiveness of measles vaccination and vitamin a treatment', The International Journal of Epidemiology, Vol. 39, No. 1, pp.i48-i55.

Szabo, G. and Toke, C. (1998) 'Evolutionary prisoner's dilemma game on a square lattice', Physical Review E, Vol. 58, No. 1, pp.69-73.

Traulsen, A., Pacheco, J.M. and Nowak, M.A. (2007) 'Pairwise comparison and selection temperature in evolutionary game dynamics', Journal of Theoretical Biology, Vol. 246, No. 3 , pp.522-529.

Vardavas, R., Breban, R. and Blower, S. (2007) 'Can influenza epidemics be prevented by voluntary vaccination?', PLoS Computational Biology, Vol. 3, No. 5, p.e85.

Wang, Z., Kokubo, S., Tanimoto, J., Fukuda, E. and Shigaki, K. (2013) 'Insight into the so-called spatial reciprocity', Physical Review E, Vol. 88, No. 4, p.042145. 


\section{Supplementary material}

\section{Disease propagation in social networks}

Figure 8 shows disease propagation in lattice populations and the Barabási-Albert scale-free (BA-SF) network, obtained through computational simulation. Each plotted point represents an average over 100 runs. As can be seen in this figure, for lattice populations, in the case of low $\beta$ values, disease propagation is significantly inhibited by the local spatial clustering effect (Fu et al., 2011). On the other hand, unlike with lattice populations, a BA-SF network has a very sensitive $\beta$, and infectious diseases can easily spread due to the presence of degree heterogeneity (Keeling and Eames, 2005; Pastor-Satorras and Vespignani, 2001).

Figure 8 Final epidemic sizes as a function of disease transmission rate $\beta$, when no individuals are vaccinated in each network

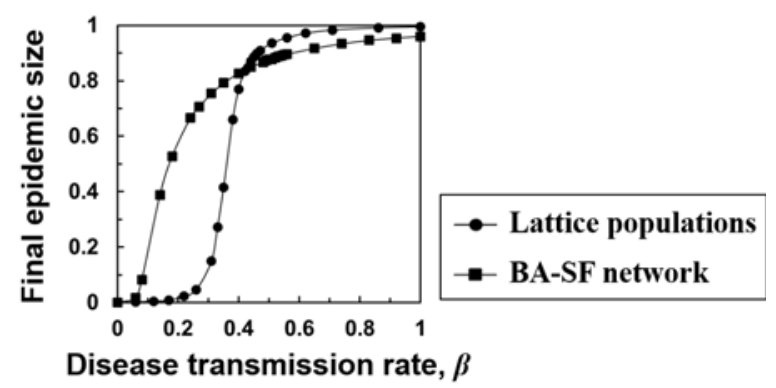

